\title{
Cervical Disc Replacement: Trends, Costs, and Complications
}

\author{
Nickul Saral Jain ${ }^{*}$, Ailene Nguyen ${ }^{*}$, Blake Formanek, Ram Alluri, Zorica Buser, Ray Hah, Jeffrey Chun Wang \\ Department of Orthopaedic Surgery, Keck School of Medicine, University of Southern California, Los Angeles, CA, USA
}

Study Design: Retrospective review of insurance database.

Purpose: To investigate national trends, complications, and costs after cervical disc replacement (CDR) using an administrative insurance database representative of the United States population.

Overview of Literature: As CDR continues to be used to treat patients with cervical stenosis, it is important to gain a better understanding of its use on a national level, potential complications, and cost. This information will allow for optimal patient counseling, risk stratification, and healthcare cost assessments. Several prior studies have investigated complications associated with CDR, but they have been limited by small sample size, single institution experiences, limited follow-up, and potential conflicts of interest. Methods: Patients who underwent single or multilevel CDR between 2007 and 2015 were identified using an insurance database. We collected data on annual trends, reimbursement costs, patient demographic information, hospital information, and information on complications from the time of operation to 1 year postoperative.

Results: Total of 293 patients underwent either single or multilevel CDR. The number of procedures increased nonlinearly over time at an average of $17 \%$ per year, with a greater increase seen in the outpatient setting. Less than $3.7 \%$ of patients had new onset pain within 1 year after CDR. Within 1 year, 12.3\% of patients reported a mechanical and/or bone-related complication. There were no patients who indicated a new nerve injury within 6 months of follow-up. Less than $3.7 \%$ of patients presented with dysphagia or dysphonia within 6 months, infection within 3 months, or a revision or reoperation within 1 year. Average reimbursement for single-level inpatient versus outpatient CDR was US $\$ 33,696.28$ and US $\$ 34,675.12$, respectively $(p=0.29$ ).

Conclusions: This study demonstrated that the use of CDR continued to increase. The most common complication was mechanical and/or bone-related, and cost analysis demonstrated no significant difference between inpatient and outpatient CDR.

Keywords: Cervical disc arthroplasty; Complications; Costs and cost analysis; Demography

\section{Introduction}

Cervical spondylosis is a highly variable, progressive disease that may result in stenosis secondary to disc herniation, disc degeneration, osteophyte formation, or hypertrophy of ligamentous structures. Patients with symptomatic cervical spondylosis may present with any combination of axial neck pain, radiculopathy, or myelopathy. Initial conservative management may include lifestyle modification, anti-inflammatory medications,

Received Aug 3, 2019; Revised Sep 23, 2019; Accepted Nov 12, 2019

Corresponding author: Zorica Buser

Department of Orthopaedic Surgery, Keck School of Medicine, University of Southern California, 1450 San Pablo St, HC4 - \#5400A, Los Angeles, CA, 90033, USA

Tel: +1-323-442-0206, Fax: +1-323-442-6990, E-mail: zbuser@usc.edu

*These authors contributed equally to this work. 
physical therapy, epidural steroid injections, or possible temporary immobilization. Operative intervention may be indicated for patients with persistent symptoms that are refractory to conservative management.

A myriad of surgical techniques is available to decompress cervical stenosis. These range from anterior to posterior approaches with or without fusion. The optimal surgical intervention is still a matter of debate, and the choice of intervention is largely dependent on the anatomic structures causing the resultant stenosis, the number of cervical levels involved, patient-related factors, and surgeon preference. Historically, anterior cervical discectomy and fusion (ACDF) has resulted in excellent outcomes and is the most common surgical treatment for cervical radiculopathy and/or myelopathy [1]. Although the success of ACDF is well documented, it relies on fusion of the involved cervical levels. This leads to increased load transmission and hypermobility at adjacent levels, which predisposes these levels to adjacent segment degeneration (ASD) [2]. Resultant symptomatic ASD may be present in up to $25 \%$ of patients at 10 years, and the additional pathology cephalad or caudad to the index fused cervical segment may negate initially good outcomes and necessitate revision decompression and possible further extension of the fused segment(s) [3-5].

Cervical disc replacement (CDR) is a technique developed to maintain disc space height and motion after discectomy to prevent abnormal load transmission at adjacent segments, which would hypothetically decrease the risk for ASD. In vitro results have demonstrated that adjacent segment motion, intradiscal pressure, and joint loading are unchanged after CDR [6]. CDR was initially approved by the Food and Drug Administration in 2007 when initial and subsequent studies demonstrated overall noninferiority to ACDF [7-13]. Over the past decade, CDR has gained popularity among some spine surgeons and has become a viable alternative to ACDF, with some newer data supporting midterm reduction in adjacent segment pathology [14].

Because CDR continues to be used by spine surgeons when treating patients with cervical stenosis, it is important to gain a better understanding of its use on a national level, potential complications, and cost. This will allow for optimal patient counseling, risk stratification, and healthcare cost assessments.

Several studies have investigated complications associated with CDR, but they have been limited by small sample size, single institution experiences, limited follow-up, and potential conflicts of interest $[15,16]$. The purpose of this study was to investigate national trends, complications, and costs after CDR using an administrative insurance database that allows for longitudinal tracking of patients and captures a demographic that is generally representative of the population of the United States.

\section{Materials and Methods}

\section{Patients}

We performed a retrospective review of the Humana subset of the PearlDiver Patient Record Database (PearlDiver Inc., Warsaw, IN, USA). This commercially available database consists of 20 million patient records from the Humana national health insurance provider from 2007 to 2016. It spans all age groups and represents geographic areas throughout the entire United States. The database allows for the sorting of clinical diagnoses by International Classification of Diseases (ICD) and Current Procedural Terminology (CPT) codes.

Patients undergoing CDR between January 1, 2007 and September 30, 2015 were identified using CPT codes. We collected data on annual trends, reimbursement costs, and patient demographic information, including sex, age, and inpatient or outpatient status. Patients' data were collected from the time of operation to 1-year postoperation. Common complications were identified and queried in the database using CPT, ICD-9, and ICD-10 codes (Table 1). Patients were followed for 3 months to 1 year following the index procedure to determine if first-instance complications arose. We grouped common complications into seven categories (Table 1). Due to privacy limitations, PearlDiver does not release the exact number of patients if there are fewer than 11 patients in any category.

The seven complications were pain, those that were mechanical and bone-related, nerve injury, dysphagia and dysphonia, infections, adverse reactions, and revision and reoperations.

The pain category included myelopathy, radiculopathy, arm, neck, and pain due to the implant. Follow-up related to pain lasted for 1-year postprocedure. Pain was separated as either the first instance coded after surgery or as continued pain that had been coded prior to surgery and continued postoperatively. Mechanical and bone-related complications included malpositioning of the implant, 
Table 1. Procedural and diagnostic codes used to identify patients in the PearlDiver database

\begin{tabular}{|c|c|}
\hline Description & Code \\
\hline \multicolumn{2}{|l|}{ Cervical disc replacement } \\
\hline Single-level & СРТ-22856 \\
\hline Multi-level & СРТ-22858 \\
\hline Pain & $\begin{array}{l}\text { ICD-9-D-7231, ICD-9-D-7234, ICD-9-D-3369, ICD-9-D-72271, ICD-9-D-99675, ICD-9-D-99679, ICD-9-D-7295, ICD-10-D-M542, } \\
\text { ICD-10-D-M5412, ICD-10-D-M5413, ICD-10-D-G959, ICD-10-D-M5000, ICD-10-D-T8584, ICD-10-D-M79601:M79603, ICD-10- } \\
\text { D-M79621, ICD-10-D-M79622, ICD-10-D-M79629, ICD-10-D-M79631, ICD-10-D-M79632, ICD-10-D-M79639 }\end{array}$ \\
\hline Dysphagia \& dysphonia & ICD-9-D-78720, ICD-9-D-78729, ICD-9-D-78442, ICD-10-D-R1310, ICD-10-D-R1319, ICD-10-D-R490 \\
\hline Nerve injury & $\begin{array}{l}\text { ICD-9-D-9517, ICD-9-D-9530, ICD-9-D-9073, ICD-9-D-99812, ICD-9-D-8068, ICD-9-D-8069, ICD-9-D-9529, ICD-9-D-9072, ICD- } \\
\text { 9-D-34931, ICD-9-D-34939, ICD-9-D-34440:34442, ICD-9-D-3442, ICD-10-D-S0489, ICD-10-D-S142, ICD-10-D-M96840, ICD- } \\
\text { 10-D-M96841, ICD-10-D-S14101:S14109, ICD-10-D-G9741, ICD-10-D-G9611, ICD-10-D-G8320:G8322, ICD-10-D-G8623, ICD- } \\
\text { 10-D-G8624, ICD-10-D-G830 }\end{array}$ \\
\hline Mechanical \& bone-related & $\begin{array}{l}\text { ICD-9-D-99659, ICD-9-D-V454, ICD-9-D-72810, ICD-9-D-72819, ICD-9-D-72813, ICD-9-D-99799, ICD-9-D-99640:99646, ICD- } \\
\text { 9-D-99649, ICD-10-D-T85625, ICD-10-D-T85628, ICD-10-D-M960, ICD-10-D-M679, ICD-10-D-M6150, ICD-10-D-M6158, ICD- } \\
\text { 10-D-M5030, ICD-10-D-M9689, ICD-10-D-T84498, ICD-10-D-T84039, ICD-10-D-T84029, ICD-10-D-T84019, ICD-10-D-T84049, } \\
\text { ICD-10-D-T84059, ICD-10-D-T84069, ICD-10-D-T84119, ICD-10-D-T84129, ICD-10-D-T84199, ICD-10-D-T84498 }\end{array}$ \\
\hline Infection & $\begin{array}{l}\text { ICD-9-D-99851, ICD-9-D-99859, ICD-9-D-99660, ICD-9-D-99663, ICD-9-D-99666, ICD-9-D-99669, ICD-9-D-99667, ICD-9-D-99677, } \\
\text { ICD-9-D-99678, ICD-10-D-T81.40:T81.43, ICD-10-D-T84.48, ICD-10-D-T84.50, ICD-10-D-T85.79, ICD-10-D-T84.7, ICD-10-D- } \\
\text { T84.9, ICD-10-D-T84.60, ICD-10-D-T84.63 }\end{array}$ \\
\hline Adverse reaction & $\begin{array}{l}\text { ICD-9-D-99811, ICD-9-D-99812, ICD-10-D-M96830, ICD-10-D-M96831, ICD-10-D-T8481:T8483, ICD-10-D-T8485, ICD-10-D- } \\
\text { T8586 }\end{array}$ \\
\hline Revisions \& reoperations & $\begin{array}{l}\text { CPT-22864, CPT-22554, CPT-22220, CPT-22861, CPT-63075, ICD-9-P-8009, ICD-9-P-8102, ICD-9-P-8101, ICD-9-P-7739, ICD- } \\
\text { 9-P-8466, ICD-9-P-8051 }\end{array}$ \\
\hline
\end{tabular}

CPT, Current Procedural Terminology; ICD, International Classification of Diseases.

pseudarthrosis, heterotrophic ossification, and adjacent disc degeneration for up to 1 year postoperatively. The nerve injury category included nerve root injury, cervical cord injury, dural tear, and C5 palsy for up to 6 months postoperatively. Overall and cervical spine-specific dysphagia and dysphonia were grouped together into one category and followed for 6 months. The infection category included superficial, deep, wound, surgical site, and device-related infections within 3 months postoperatively. Adverse reactions included hemorrhage, embolism, fibrosis, stenosis, and thrombosis within 3 months of surgery. Revision and reoperation procedures were followed for 1 year and included removal of CDR, conversion to ACDF, osteotomy, revision, and cervical discectomy with decompression.

Patient data were completely deidentified and therefore our study did not require institutional review board approval.

\section{Statistical analysis}

Patients were grouped by age into 5-year increments from 10 to $\geq 90$ years. Incidence was calculated as procedures per 100,000 members. The charge information we used was calculated as amount billed by the institution for each patient for care surrounding the index procedure. $T$-tests were used to calculate $p$-values to compare rates, and statistically significant $p$-values were defined as $p<0.05$.

\section{Results}

\section{Demographic trends}

We studied 293 patients who received an index CDR between 2009 and 2015. The number of procedures increased nonlinearly over time at an average of $17 \%$ per year (Fig. 1, Table 2). Eleven multilevel CDR procedures were performed, all of them in 2015 . The number of outpatient procedures increased over time, with $68 \%$ of CDR procedures performed in the outpatient setting in 2015 (Table 3). CDR was most commonly performed in patients aged 40 to 54 years old, and $50.1 \%$ of patients were female (Table 2). The database reflected information on patients from much of the United States, with $71 \%$ of the CDR procedures performed in the South, $16.4 \%$ in the Midwest, $11.3 \%$ in the West, and $<3.7 \%$ in the Northeast (Table 2). 


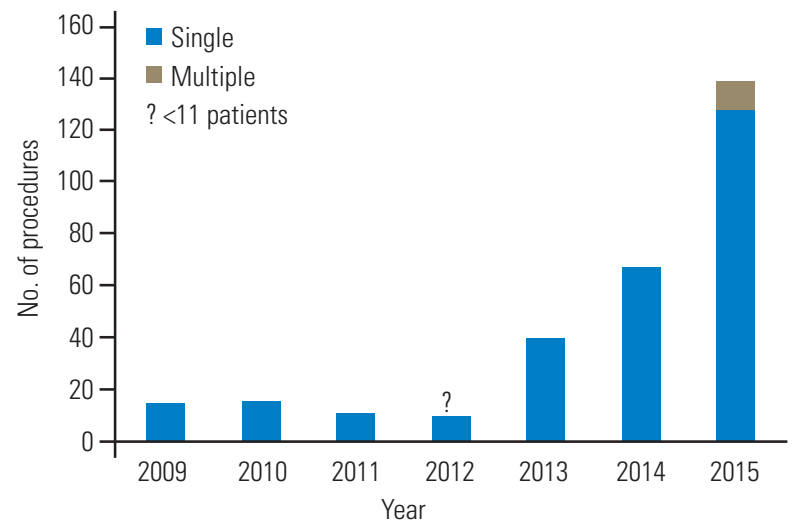

Fig. 1. Breakdown of cervical disc replacement procedures by number of contiguous levels treated. A question mark indicates fewer than 11 patients, where an exact number could not be obtained due to PearlDiver limitations on privacy.

Table 2. Demographics of patients undergoing cervical disc replacement within the Humana database

\begin{tabular}{|cc|}
\hline Characteristic & No. of patients $(\%)$ \\
\hline Sex & $147(50.1)$ \\
\hline Female & $146(49.9)$ \\
\hline Male & \\
\hline Age (yr) & $<11(<3.7)$ \\
\hline $25-29$ & $15(5.1)$ \\
\hline $30-34$ & $34(11.6)$ \\
\hline $35-39$ & $57(19.4)$ \\
\hline $40-44$ & $60(20.5)$ \\
\hline $45-49$ & $54(18.4)$ \\
\hline $50-54$ & $28(9.6)$ \\
\hline $55-59$ & $23(7.8)$ \\
\hline $60-64$ & $11(3.7)$ \\
\hline $65-69$ & $<11(<3.7)$ \\
\hline $70-74$ & 293 \\
\hline Year of procedure & $208(71.0)$ \\
\hline 2009 & $15(5.1)$ \\
\hline 2010 & $16(5.5)$ \\
\hline 2011 & $11(3.7)$ \\
\hline 2012 & $<11(<3.7)$ \\
\hline 2013 & $40(36.2)$ \\
\hline 2014 & $67(22.8)$ \\
\hline 2015 & $139(47.4)$ \\
\hline Region & $28(16.4)$ \\
\hline Midwest & \\
\hline Northeast & \\
\hline
\end{tabular}

Table 3. Inpatient and outpatient status for patients undergoing cervical disc replacement by year

\begin{tabular}{lccc} 
& \multicolumn{3}{c}{ Year } \\
\cline { 2 - 4 } Procedure type & 2013 & 2014 & 2015 \\
Inpatient & 23 & 26 & 47 \\
Outpatient & 21 & 44 & 99 \\
Total & 44 & 70 & 146 \\
\hline
\end{tabular}

Table 4. Overall complication rates for cervical disc replacement

\begin{tabular}{lc} 
Complications & No. of patients $(\%)$ \\
\hline Pain & \\
\hline \multicolumn{1}{c}{ First instance } & $<11(<3.7)$ \\
\hline \multicolumn{1}{c}{ Continued } & $218(74.4)$ \\
\hline Mechanical and bone-related & $36(12.3)$ \\
\hline Dysphagia/dysphonia & $<11(<3.7)$ \\
\hline Infection & $<11(<3.7)$ \\
\hline Adverse reaction & $<11(<3.7)$ \\
\hline Revision or reoperation & $<11(<3.7)$ \\
\hline Nerve injury & 0 \\
\hline Total surgeries & 293 \\
\hline
\end{tabular}

\section{Complications}

Fewer than $3.7 \%$ of patients $(<11)$ reported new pain within 1 year after CDR, and $74.4 \%$ of patients (218) had continued pain within 1 year after CDR. Within 1 year, $12.3 \%$ (36) of patients reported a mechanical and/ or bone-related complication. No patients had nerve injury within 6 months after CDR. Fewer than 11 patients $(<3.7 \%)$ presented with dysphagia or dysphonia within 6 months, an infection within 3 months, an adverse reaction within 3 months, or a revision or reoperation within 1 year (Table 4). Due to PearlDiver limitations on privacy, exact numbers could not be obtained.

\section{Cost analysis}

For all 293 CDR procedures and complications, the mean reimbursement cost was US $\$ 35,711.94$. The mean cost was US $\$ 34,675.12$ for single-level procedures and US $\$ 62,292$ for multiple-level procedures (Table 5). CDR with a first instance of pain after surgery averaged a higher cost at US $\$ 50,768.80$ compared with the mean cost of US 
Table 5. Total reimbursements for patients undergoing cervical disc replacement

\begin{tabular}{|c|c|c|c|}
\hline Variable & Total (US \$) & Mean \pm standard deviation (US \$) & No. of patients \\
\hline \multicolumn{4}{|l|}{ Levels } \\
\hline Single & $9,778,385.00$ & $34,675.12 \pm 30,341.35$ & 282 \\
\hline Multiple & $685,213.00$ & $62,292.09 \pm 28,527.74$ & 11 \\
\hline \multicolumn{4}{|l|}{ Complications } \\
\hline \multicolumn{4}{|l|}{ Pain } \\
\hline First instance & $253,844.00$ & $50,768.80 \pm 33,396.68$ & $<11$ \\
\hline Continued & $8,323,331.00$ & $38,180.42 \pm 32,268.60$ & 218 \\
\hline Mechanical and bone-related & $1,717,647.00$ & $47,712.42 \pm 49,494.71$ & 36 \\
\hline Dysphagia/dysphonia & $284,350.00$ & $31,594.44 \pm 29,366.38$ & $<11$ \\
\hline Infection & $55,511.00$ & $18,503.67 \pm 8,131.62$ & $<11$ \\
\hline Adverse reaction & $52,698.00$ & $52,698.00$ & $<11$ \\
\hline Revision or reoperation & $363,099.00$ & $90,774.75 \pm 104,107.41$ & $<11$ \\
\hline Nerve injury & 0 & 0 & 0 \\
\hline Overall & $10,463,598.00$ & $35,711.94 \pm 30,682.92$ & 293 \\
\hline
\end{tabular}

$\$ 38,180.42$ for patients who had continued pain. This was a nonsignificant difference $(p>0.05)$ (Table 5). There was also no significant difference between the overall mean cost for all CDR procedures compared to the mean cost for first instance of pain $(p>0.05)$. However, there was a significantly higher cost for patients with continued pain when compared to the overall mean $(p<0.05)$. Mechanical and/or bone-related complication reimbursements had a mean cost of US $\$ 47,712.42$. The dysphagia and dysphonia reimbursement mean cost was US $\$ 31,594.44$, while wound infections had a mean cost of US $\$ 18,503.67$. Adverse reactions had a mean reimbursement cost of US \$52,698, and revisions and reoperations incurred a total mean cost of US $\$ 90,774.75$. Total reimbursements, means, and standard deviations can be found in Table 5 .

\section{Discussion}

CDR is a relatively new treatment option that allows for effective discectomy, restoration of disc space height, and decompression of spinal stenosis while preserving range of motion for the patient. Well-designed studies have demonstrated no inferiority to ACDF, and even superior outcomes in patients with select indications, which has led to increased acceptance and popularity of the procedure [7-13]. Prior studies demonstrated significant increases in CDR utilization from 2006 to 2009, followed by a plateau and slight decrease in use relative to ACDF procedures in more recent years [14-17]. The results of this study differ from prior studies in that the use of CDR continued to increase nonlinearly at a rate of $17 \%$ per year from 2009 to 2015 without evidence of a plateau effect in the 7 years assessed. This may be due to the differing nature of the databases being used. The highest percentage of patients who underwent CDR were between ages 40 to 54 years old and approximately 50\% were female, similar to other large database studies [18-20].

The most common complication (found in $12.3 \%$ of patients) in the first postoperative year after CDR was mechanical and/or bone-related, which included malpositioning of the implant, heterotrophic ossification, and adjacent disc degeneration. However, due to the limitations of the database data, we could not calculate the exact incidence of component malpositioning or migration, as these complications are often grouped together with other similar complications due to the overall low incidence. Another limitation was that we did not have information on the extent of ASD and whether it was symptomatic or asymptomatic.

In our study, less than $3.7 \%$ of patients had a revision or reoperation within 1 year. Zhong et al. [21], in a metaanalysis, calculated a similar reoperation rate of $4 \%$ due to device malposition, migration, subsidence, or recurring pain after CDR. In a second meta-analysis, a 7.4\% incidence of neck pain, implant migration, or radiculopathy was described at 4 years postoperatively [22]. 
The incidence of heterotopic ossification (HO) was less than $12.3 \%$ in the current study. $\mathrm{HO}$ is thought to be a dynamic, progressive phenomenon, and therefore its incidence is likely affected by the length of follow-up. Two prior studies that have assessed $\mathrm{HO}$ at 1-year follow-up after $\mathrm{CDR}$ have described much higher rates of $\mathrm{HO}$, ranging from $17.8 \%$ to $44.6 \%[23,24]$. The high rate $(74.4 \%)$ of continued pain after CDR noted in our study is unlikely to be continued postoperative pain but rather a result of providers continuing to code preoperative radiculopathy or cervicalgia during postoperative follow-up. The constraints of the database study limit us from fully elucidating this point, but when we go by this assumption, we can conclude that costs were higher for patients who had a new incidence of pain within 1 year after CDR.

Dysphagia was present in $<3.7 \%$ of patients at 6 months after CDR. In previous studies, the incidence of dysphagia has tended to decrease with time. One study noted a $37 \%$ incidence at 1 week, $7 \%$ incidence at 6 months, and 6\% incidence at 12 months postoperatively [25]. Other studies have demonstrated an approximate $11 \%$ incidence of dysphagia at 24 to 84 months after CDR $[10,26]$. It is unclear why an increasing rate of dysphagia was noted with longer follow-up in our current study. No nerve injuries (0\%) were noted at 6 months in the current study. Previous studies have demonstrated a $0.9 \%$ to $4.1 \%$ neurologic complication rate anywhere from 2 to 7 years after CDR [8,26-28]. Infection was also present in $<3.7 \%$ of patients within 3 months after CDR. This result is consistent with prior studies in the literature that documented infection rates ranging from $0 \%$ to $2.9 \%$ [8,26,28-30]. Lastly, reoperation or revision within 1 year after CDR occurred in $<3.7 \%$ of patients. Other large database studies have demonstrated similarly low reoperation rates of $<1 \%$, though these studies only assessed revisions within 30 days [30].

The last aim of our study was to assess the cost associated with CDR in an administrative insurance database. The mean reimbursement cost was US $\$ 34,675.12$ for single-level procedures and US $\$ 62,292$ for multiplelevel procedures. When including the cost of associated complications, the mean reimbursed cost increased to US $\$ 35,711.94$. Cost in the PearlDiver database is determined based on the amount billed by an institution for care surrounding or related to the CDR. A study by Saifi et al. [14] determined the mean cost of CDR to be US $\$ 13,197$, which was calculated as the amount billed by the hospital for the procedure, excluding physician fees. The study of
Saifi et al. [14] used the National Inpatient Sample, which only allows the assessment of inpatient data. This partially explains the lower cost documented in their study compared to the present study, which captures both inpatient and outpatient cost data. A study by Radcliff et al. [19] utilized the Blue Health Intelligence database and demonstrated a cost nearly identical to the cost we determined to be associated with CDR, US $\$ 34,979$.

The results of this study must be interpreted within the limitations of its design. First, a large-scale administrative database was utilized and the accuracy of the results is subject to coding error, particularly when assessing complications. Second, we only assessed postoperative complications from 3 to 12 months after CDR and thereby may be underestimating the true incidence of complications related to CDR. Additionally, PearlDiver does not release the exact number of patients if there are fewer than $11 \mathrm{pa}-$ tients in any category, and we thus had to combine similar complications into groups of related complications and we could not do further subanalysis. Lastly, the PearlDiver database is an insurance database and is not designed to be a true representative sample of the US population. Therefore, the results of this study may not be generalizable to the US population at large.

Although there are limitations to our study, there are significant strengths. First is our use of a large national database that broadly represents the US population. Second, the database allows for the longitudinal tracking of patients across the inpatient and outpatient settings, and so complications and cost are captured in both settings. Lastly, the PearlDiver database is 1 of only a few databases that allows for identification of patients using both CPT and ICD codes, allowing for more accurate identification of a particular cohort.

\section{Conclusions}

This study demonstrates that the use of CDR continues to increase in a nonlinear fashion, which contrasts with prior studies demonstrating a plateau or recent relative decrease in CDR. The most common complication, found in $12.3 \%$ of patients, was mechanical and/or bone-related, which is slightly higher than what was previously documented in other studies. Complications related to dysphagia had a lower incidence in this study compared to previously reported data. Nerve injury, infection, and reoperation occurred at a rate similar to that seen in other large database 
studies. Lastly, the cost of CDR calculated in this study was nearly identical to a prior study using an insurance database, further validating those results and lending evidence as to why these numbers should be used in future studies comparing the cost of CDR with the cost of ACDF.

\section{Conflict of Interest}

No potential conflict of interest relevant to this article was reported.

\section{References}

1. Smith GW, Robinson RA. The treatment of certain cervical-spine disorders by anterior removal of the intervertebral disc and interbody fusion. J Bone Joint Surg Am 1958;40-A:607-24.

2. Elsawaf A, Mastronardi L, Roperto R, Bozzao A, Caroli M, Ferrante L. Effect of cervical dynamics on adjacent segment degeneration after anterior cervical fusion with cages. Neurosurg Rev 2009;32:215-24.

3. Hilibrand AS, Carlson GD, Palumbo MA, Jones PK, Bohlman HH. Radiculopathy and myelopathy at segments adjacent to the site of a previous anterior cervical arthrodesis. J Bone Joint Surg Am 1999;81:51928.

4. Robertson JT, Papadopoulos SM, Traynelis VC. Assessment of adjacent-segment disease in patients treated with cervical fusion or arthroplasty: a prospective 2-year study. J Neurosurg Spine 2005;3:41723.

5. Gore DR, Sepic SB. Anterior discectomy and fusion for painful cervical disc disease: a report of $50 \mathrm{pa}-$ tients with an average follow-up of 21 years. Spine (Phila Pa 1976) 1998;23:2047-51.

6. Dmitriev AE, Cunningham BW, Hu N, Sell G, Vigna F, McAfee PC. Adjacent level intradiscal pressure and segmental kinematics following a cervical total disc arthroplasty: an in vitro human cadaveric model. Spine (Phila Pa 1976) 2005;30:1165-72.

7. Gornet MF, Lanman TH, Burkus JK, et al. Cervical disc arthroplasty with the Prestige LP disc versus anterior cervical discectomy and fusion, at 2 levels: results of a prospective, multicenter randomized controlled clinical trial at 24 months. J Neurosurg Spine 2017;26:653-67.

8. Janssen ME, Zigler JE, Spivak JM, Delamarter RB,
Darden BV 2nd, Kopjar B. ProDisc-C total disc replacement versus anterior cervical discectomy and fusion for single-level symptomatic cervical disc disease: seven-year follow-up of the prospective randomized U.S. Food and Drug Administration Investigational Device Exemption Study. J Bone Joint Surg Am 2015;97:1738-47.

9. Radcliff K, Davis RJ, Hisey MS, et al. Long-term evaluation of cervical disc arthroplasty with the Mobi-C(c) cervical disc: a randomized, prospective, multicenter clinical trial with seven-year follow-up. Int J Spine Surg 2017;11:31.

10. Burkus JK, Traynelis VC, Haid RW Jr, Mummaneni PV. Clinical and radiographic analysis of an artificial cervical disc: 7-year follow-up from the Prestige prospective randomized controlled clinical trial: clinical article. J Neurosurg Spine 2014;21:516-28.

11. Vaccaro A, Beutler W, Peppelman W, et al. Clinical outcomes with selectively constrained SECURE-C cervical disc arthroplasty: two-year results from a prospective, randomized, controlled, multicenter investigational device exemption study. Spine (Phila Pa 1976) 2013;38:2227-39.

12. Heller JG, Sasso RC, Papadopoulos SM, et al. Comparison of BRYAN cervical disc arthroplasty with anterior cervical decompression and fusion: clinical and radiographic results of a randomized, controlled, clinical trial. Spine (Phila Pa 1976) 2009;34:101-7.

13. Phillips FM, Geisler FH, Gilder KM, Reah C, Howell KM, McAfee PC. Long-term outcomes of the US FDA IDE prospective, randomized controlled clinical trial comparing PCM cervical disc arthroplasty with anterior cervical discectomy and fusion. Spine (Phila Pa 1976) 2015;40:674-83.

14. Saifi C, Fein AW, Cazzulino A, et al. Trends in resource utilization and rate of cervical disc arthroplasty and anterior cervical discectomy and fusion throughout the United States from 2006 to 2013. Spine J 2018;18:1022-9.

15. Ioannidis JP. Adverse events in randomized trials: neglected, restricted, distorted, and silenced. Arch Intern Med 2009;169:1737-9.

16. Narain AS, Hijji FY, Yom KH, Kudaravalli KT, Singh K. Cervical disc arthroplasty: do conflicts of interest influence the outcome of clinical studies? Spine J 2017;17:1026-32.

17. Lu Y, McAnany SJ, Hecht AC, Cho SK, Qureshi SA. 
Utilization trends of cervical artificial disc replacement after FDA approval compared with anterior cervical fusion: adoption of new technology. Spine (Phila Pa 1976) 2014;39:249-55.

18. Bhashyam N, De la Garza Ramos R, Nakhla J, et al. Thirty-day readmission and reoperation rates after single-level anterior cervical discectomy and fusion versus those after cervical disc replacement. Neurosurg Focus 2017;42:E6.

19. Radcliff K, Zigler J, Zigler J. Costs of cervical disc replacement versus anterior cervical discectomy and fusion for treatment of single-level cervical disc disease: an analysis of the Blue Health Intelligence database for acute and long-term costs and complications. Spine (Phila Pa 1976) 2015;40:521-9.

20. Rumalla K, Smith KA, Arnold PM. Cervical total disc replacement and anterior cervical discectomy and fusion: reoperation rates, complications, and hospital resource utilization in 72688 patients in the United States. Neurosurgery 2018;82:441-53.

21. Zhong ZM, Zhu SY, Zhuang JS, Wu Q, Chen JT. Reoperation after cervical disc arthroplasty versus anterior cervical discectomy and fusion: a meta-analysis. Clin Orthop Relat Res 2016;474:1307-16.

22. Wu TK, Liu H, Wang BY, Meng Y. Minimum fouryear subsequent surgery rates of cervical disc replacement versus fusion: a meta-analysis of prospective randomized clinical trials. Orthop Traumatol Surg Res 2017;103:45-51.

23. Leung C, Casey AT, Goffin J, et al. Clinical significance of heterotopic ossification in cervical disc replacement: a prospective multicenter clinical trial. Neurosurgery 2005;57:759-63.

24. Chen J, Wang X, Bai W, Shen X, Yuan W. Prevalence of heterotopic ossification after cervical total disc arthroplasty: a meta-analysis. Eur Spine J 2012;21:67480.

25. Yang Y, Ma L, Liu H, et al. Comparison of the incidence of patient-reported post-operative dysphagia between ACDF with a traditional anterior plate and artificial cervical disc replacement. Clin Neurol Neurosurg 2016;148:72-8.

26. Anderson PA, Sasso RC, Riew KD. Comparison of adverse events between the Bryan artificial cervical disc and anterior cervical arthrodesis. Spine (Phila Pa 1976) 2008;33:1305-12.

27. Pickett GE, Sekhon LH, Sears WR, Duggal N. Complications with cervical arthroplasty. J Neurosurg Spine 2006;4:98-105.

28. Nandyala SV, Marquez-Lara A, Fineberg SJ, Singh $\mathrm{K}$. Comparison of revision surgeries for one- to twolevel cervical TDR and ACDF from 2002 to 2011. Spine J 2014;14:2841-6.

29. Skeppholm M, Lindgren L, Henriques T, Vavruch L, Lofgren H, Olerud C. The Discover artificial disc replacement versus fusion in cervical radiculopathy: a randomized controlled outcome trial with 2-year follow-up. Spine J 2015;15:1284-94.

30. Segal DN, Wilson JM, Staley C, Yoon ST. Outpatient and inpatient single-level cervical total disc replacement: a comparison of 30-day outcomes. Spine (Phila Pa1976) 2019;44:79-83. 